\title{
Efficacy of orlistat in non-alcoholic fatty liver disease: A systematic review and meta-analysis
}

\author{
$\mathrm{HU}_{\text {WANG }}{ }^{*}$, LI WANG $^{1 *}$, YUJIA CHENG $^{1 *}, \mathrm{ZHIQING} \mathrm{XIA}^{1}$, YIFENG LIAO $^{1}$ and JIANG CAO ${ }^{2}$ \\ ${ }^{1}$ Department of Clinical Medicine, Xuzhou Medical University, Xuzhou, Jiangsu 221004; ${ }^{2}$ Department of Hematology, \\ Affiliated Hospital of Xuzhou Medical University, Xuzhou, Jiangsu 221002, P.R. China
}

Received December 14, 2017; Accepted May 10, 2018

DOI: $10.3892 /$ br.2018.1100

\begin{abstract}
In the present meta-analysis, the efficacy and safety of orlistat in the treatment of non-alcoholic fatty liver (NAFLD) and non-alcoholic steatohepatitis (NASH) were evaluated. PubMed, Embase, the Cochrane Library, Web of Science, and Wan Fang data were searched for controlled trials of orlistat in patients with NAFLD or NASH, published before August 2017. Three randomized controlled trials and four single-arm trials were included. The involved participants with NAFLD or NASH (330 patients) were analyzed for clinical outcomes including alteration in hepatic histological variables and biomarkers of liver function. Improvements were observed in levels of alanine aminotransferase [standard mean difference $(\mathrm{SMD})=-1.41 ; \mathrm{P}=0.01]$, aspartate aminotransferase $(\mathrm{SMD}=-2.06 ; \mathrm{P}=0.0005), \gamma$-glutamyl transpeptidase $(\mathrm{SMD}=-1.91 ; \mathrm{P}=0.05)$, glucose [mean difference $(\mathrm{MD})=-0.51$; $\mathrm{P}=0.01]$, triglycerides $(\mathrm{MD}=-0.93 ; \mathrm{P}=0.01)$, homeostasis model assessment of insulin resistance index $(\mathrm{MD}=-1.05$; $\mathrm{P}=0.04)$ and body mass index $(\mathrm{MD}=-1.97 ; \mathrm{P}=0.02)$, but not in liver fibrosis score $(\mathrm{SMD}=-0.14 ; \mathrm{P}=0.71)$. On sub-analyses of the different patient groups, no significant differences were observed in patients with NASH. Taken together, these findings demonstrate that orlistat could serve as a therapeutic drug to improve biochemical indicators of liver damage, but not as
\end{abstract}

Correspondence to: Dr Jiang Cao, Department of Hematology, Affiliated Hospital of Xuzhou Medical University, 99 Huaihai West Road, Quanshan, Xuzhou, Jiangsu 221002, P.R. China

E-mail: hyfsh864@163.com

*Contributed equally

Abbreviations: NAFLD, non-alcoholic fatty liver; NASH, non-alcoholic steatohepatitis; ALT, alanine aminotransferase; AST, aspartate aminotransferase; GGT, $\gamma$-glutamyl transpeptidase; TG, triglyceride; HOMA-IR, homeostasis model assessment of insulin resistance; BMI, body mass index; RCT, randomized controlled trial; MD, mean difference; SMD, standard mean difference; $95 \%$ CI, $95 \%$ confidence interval

Key words: meta-analysis, non-alcoholic fatty liver disease, non-alcoholic steatohepatitis, orlistat first-choice drug for the management of NAFLD or NASH; thus suggesting a novel palliative drug only for the treatment of NAFLD.

\section{Introduction}

Non-alcoholic fatty liver disease (NAFLD) is a chronic liver disease characterized by hepatic fat accumulation in patients with little to no history of alcohol consumption (1). Up to $30 \%$ of the worldwide population is affected by NAFLD, among which populations in developed countries are particularly affected, including those in the United States $(2,3)$. The occurrence and development of NAFLD are associated with metabolic syndrome, which comprises a group of disorders including obesity, increased plasma triglycerides (TGs) and reduced insulin sensitivity (4). More specifically, Li et al (5) reported that obesity may be an independent risk factor for NAFLD. NAFLD is considered a public health concern due to its potential to progress to non-alcoholic steatohepatitis (NASH), fibrosis, cirrhosis and even hepatocellular carcinoma $(6,7)$. The primary therapy for NAFLD is lifestyle management to prevent obesity; however, long-term changes in eating habits and weight loss are difficult to achieve and maintain (8). Due to the lack of effective therapy options and consequences of disease progression, the development of novel pharmacological treatments for NAFLD should be actively pursued.

Orlistat is among the few types of over-the-counter diet pill available worldwide, and is a potent and long-lasting gastrointestinal lipase inhibitor that directly blocks intestinal absorption of dietary fat, and thus promotes weight loss $(9,10)$. In clinical trials, patients treated with orlistat exhibited lower fasting glucose, fasting insulin, TGs, insulin resistance as assessed via the homeostasis model assessment of insulin resistance (HOMA-IR) and body mass index (BMI), along with greater weight loss compared with those treated with placebo (11-13). Furthermore, orlistat was demonstrated to reduce transaminase levels (14), commonly used as biomarkers to assess liver damage $(14,15)$. As an inhibitor of gastroenteric lipase, the long-term application of orlistat is considered to have no toxic side effects (16); additionally, any adverse effects in the contexts of cardiovascular outcome and carcinogenesis are as yet unreported.

For these reasons, orlistat is expected to be an effective drug for the management of NAFLD; however, its efficacy remains controversial. Zelber-Sagi et al (17) identified that, compared 
with placebo, orlistat did not cause a significant reduction in body weight in patients with NAFLD. Thus, the purpose of the present systematic review and meta-analysis was to evaluate the efficacy and safety of orlistat in patients with NAFLD or NASH.

\section{Materials and methods}

Protocol. The meta-analysis was conducted by two investigators independently. Any differences in opinion were resolved by consensual agreement. The methods used adhered to the criteria of the Preferred Reporting Items for Systematic Reviews and Meta-Analyses guidelines (18).

Literature search strategy. In August 2017, a computer-assisted search was performed of PubMed (https://www.ncbi.nlm.nih. gov/pubmed), Embase (https://www.embase.com/\#search), Cochrane Library (https://onlinelibrary-wiley-com.webproxy. potsdam.edu/cochranelibrary/search), Web of Science (http:// isiknowledge.com) and Wan Fang data (http://www.wanfangdata. com.cn/index.html) using the following combinations of terms: 'THLP' or 'tetrahydrolipastatin' or 'Xenical' or 'Roche brand of orlistat' or 'Hoffmann-La Roche brand of orlistat' or 'Alli' or 'GlaxoSmithKline brand of orlistat' and 'non-alcoholic fatty liver disease' or 'NAFLD' or 'nonalcoholic fatty liver disease' or 'fatty liver, nonalcoholic' or 'fatty livers, nonalcoholic' or 'liver, nonalcoholic fatty' or 'livers, nonalcoholic fatty' or 'nonalcoholic fatty liver' or 'nonalcoholic fatty livers' or 'nonalcoholic steatohepatitis' or 'nonalcoholic steatohepatitides' or 'steatohepatitides, nonalcoholic' or 'steatohepatitis, nonalcoholic'.

Inclusion and exclusion criteria. Database search results were screened using the following inclusion criteria: i) NAFLD or NASH diagnosed using results of liver biopsy or imaging; ii) orlistat prescribed to patients with NAFLD or NASH; iii) if present, controls were placebo, energy-controlled diet and/or vitamin E; and iv) the study classified as a randomized controlled trial (RCT) or an observational study. Exclusion criteria were: i) The use of animal or cell models; ii) orlistat prescribed in combination with other therapeutic drugs; iii) the study classified as a review or case report; and iv) treatment of sub-cohort groups with orlistat. No language restrictions were set.

Data extraction and quality assessment. The following data were extracted from the included studies: The first author, year of publication, sample size, length of follow-up, patient characteristics (country, sex ratio, mean age and obesity status), intervention (dose, duration and diet), control (placebo or other), liver histological variables and biomarkers for liver function [alanine aminotransferase (ALT), aspartate aminotransferase (AST), $\gamma$-glutamyl transpeptidase (GGT), TGs, glucose and HOMA-IR]. Extracted data were verified by an independent investigator and any disagreements were resolved through discussion with a second investigator until a consensus was reached. The Cochrane Risk of Bias tool (19) and the Newcastle-Ottawa Quality Assessment Scale (20) were used to evaluate RCTs and observational studies, respectively. Safety was assessed based on reported treatment-related adverse events.

Statistical analysis. RevMan software (version 5.3; Cochrane, London, UK) was used for statistical analysis. Post-treatment

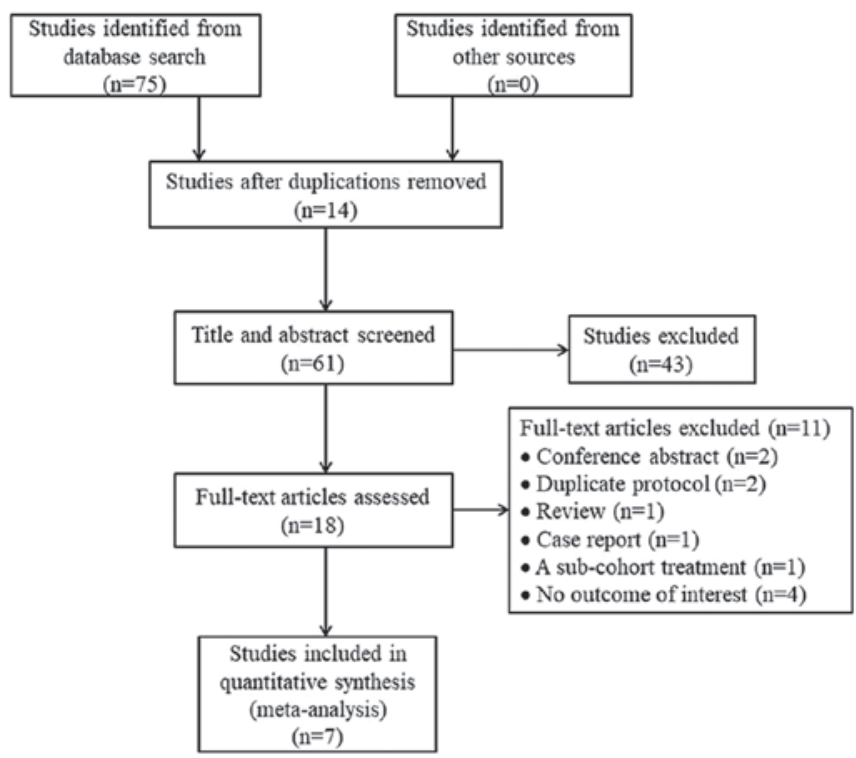

Figure 1. Flowchart of the literature search and study selection process.

and baseline values were extracted as means \pm standard deviations. For combined analyses, the mean difference (MD) or standardized mean difference (SMD) with 95\% confidence interval $(\mathrm{CI})$ to pool the extracted variables. The heterogeneity between studies was determined using the $\mathrm{I}^{2}$ statistic; $\mathrm{I}^{2}$ values of 25,50 and $75 \%$ represented low, moderate and high heterogeneity, respectively (21). In cases with $\mathrm{I}^{2}<50 \%$, a fixed-effects model was used, whereas a random-effects model was used if $\mathrm{I}^{2}>50 \%$. $\mathrm{P}<0.05$ was considered to indicate statistical significance.

\section{Results}

Literature search. The database searches yielded 75 studies, of which 14 were excluded due to duplication. Following screening of titles and abstracts for eligibility, 43 additional studies were excluded. Of the remaining 18 studies, 11 were excluded following assessment of the full-text: Two conference abstracts, two duplicate protocols, one review, one case report, one study with sub-cohort treatment with orlistat and four studies with no outcomes of interest. Ultimately, seven studies were analyzed (11-13,17,22-24) (Fig. 1).

Trial characteristics. Trial characteristics of the seven included studies are summarized in Table I. Briefly, the trials were published between 2004 and 2017 and included 330 participants. The mean age of participants ranged from 36.5 to 58.5 years, and in all but one trial, the obesity rate was $100 \%$. The duration of orlistat intervention ranged from 16 to 36 weeks, and in all studies, the dose administered was $120 \mathrm{mg}$ three times a day. The study by Fan et al (22) analyzed two treatment durations (12 and 24 weeks); due to more comprehensive reporting on the 24-week data, the 24-week group was selected for inclusion in the meta-analysis.

Quality assessment. Quality assessment of the three included RCTs $(11,17,23)$ was performed using the Cochrane Risk of Bias tool (Fig. 2). Overall, all three studies had a low risk of 


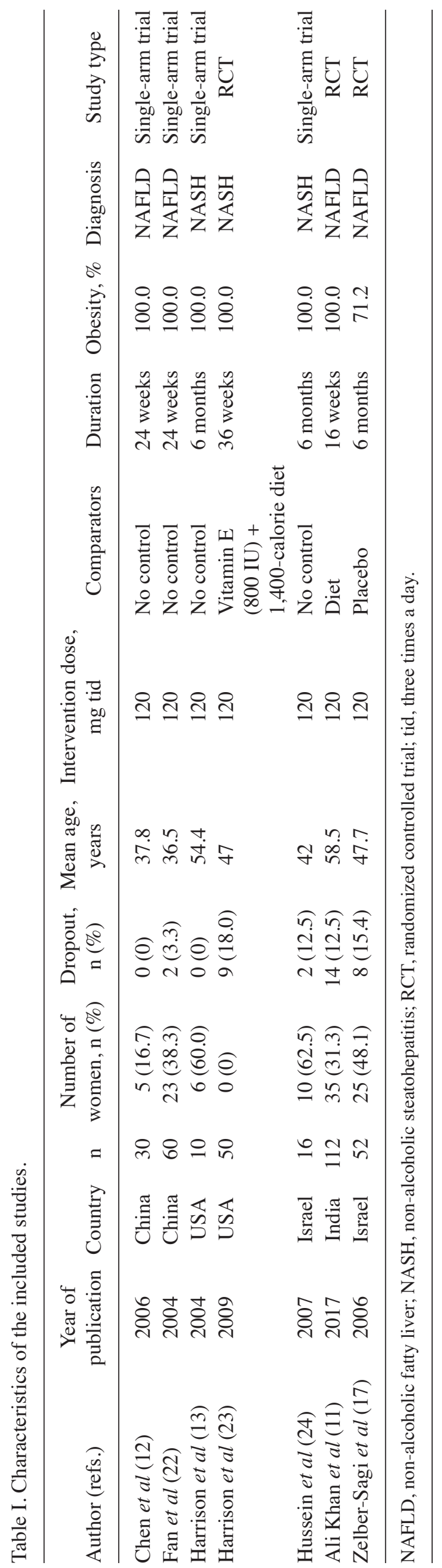

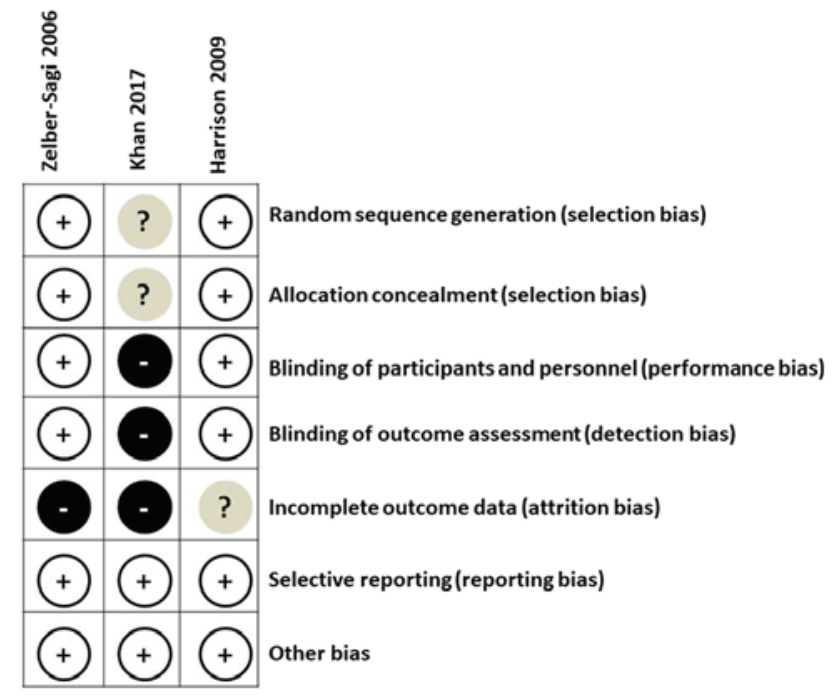

Figure 2. Summary of risk of bias assessments for the three randomized controlled trials included in the meta-analysis.

bias in selective reporting and other areas. Additionally, two of the studies $(17,23)$ had low risk of bias in random sequence generation, allocation concealment, blinding of participants and personnel, and blinding of outcome assessment. The number and cause of dropouts were identified for each study.

Quantitative data synthesis

$A L T$. ALT levels were reported in all included studies. Although between-study heterogeneity was high $\left(\mathrm{I}^{2}=95 \%\right)$, orlistat post-treatment significantly reduced ALT levels compared to baseline [SMD $=-1.41 ; 95 \%$ confidence interval $(95 \% \mathrm{CI})=-2.52$ to $-0.30 ; \mathrm{P}=0.01]$. Specific subgroup analysis indicated no significant improvement in ALT levels in patients with NAFLD (SMD $=-1.17 ; 95 \% \mathrm{CI}=-2.55$ to $0.21 ; \mathrm{I}^{2}=96 \%$; $\mathrm{P}=0.1)$ or NASH $\left(\mathrm{SMD}=-1.80 ; 95 \% \mathrm{CI}=-4.46\right.$ to $0.86 ; \mathrm{I}^{2}=96 \%$; $\mathrm{P}=0.18$; Fig. 3A).

$A S T$. AST levels were reported in six of the seven studies analyzed. Compared to baseline, orlistat treatment significantly reduced AST levels ( $\mathrm{SMD}=-2.06$; 95\% CI $=-3.23$ to -0.89 ; $\mathrm{P}=0.0005)$; however, between-study heterogeneity was high $\left(I^{2}=93 \%\right)$. In the subgroup analysis, significant difference in AST was identified in patients with NAFLD (SMD $=-2.42$; 95\% CI $=-3.95$ to $-0.90 ; \mathrm{I}^{2}=93 \% ; \mathrm{P}=0.002$ ), but not in those with NASH (SMD $=-1.75 ; 95 \% \mathrm{CI}=-4.17$ to $0.67 ; \mathrm{I}^{2}=95 \%$; $\mathrm{P}=0.16$; Fig. 3B).

GGT. The effect of orlistat on GGT levels reported in three studies was analyzed. Orlistat treatment significantly reduced GGT levels compared to baseline (SMD $=-1.91 ; 95 \% \mathrm{CI}=-3.79$ to $-0.03 ; \mathrm{P}=0.05$ ); however, between-study heterogeneity was high $\left(\mathrm{I}^{2}=96 \%\right.$; Fig. 3C).

Liver histology. In four studies included in the meta-analysis, liver biopsies were administrated on admission and iterated following the treatment with orlistat. Fibrosis was scored on a scale from 0 to 4 (25). No significant differences between post-treatment and baseline scores were observed ( $\mathrm{SMD}=-0.14$; $95 \% \mathrm{CI}=-0.88$ to $0.60 ; \mathrm{P}=0.71$ ) in patients treated with orlistat. Between-study heterogeneity was high $\left(\mathrm{I}^{2}=77 \%\right.$; Fig. 3D).

Glucose. Orlistat treatment caused significant reduction in fasting plasma glucose levels compared to baseline in patients 


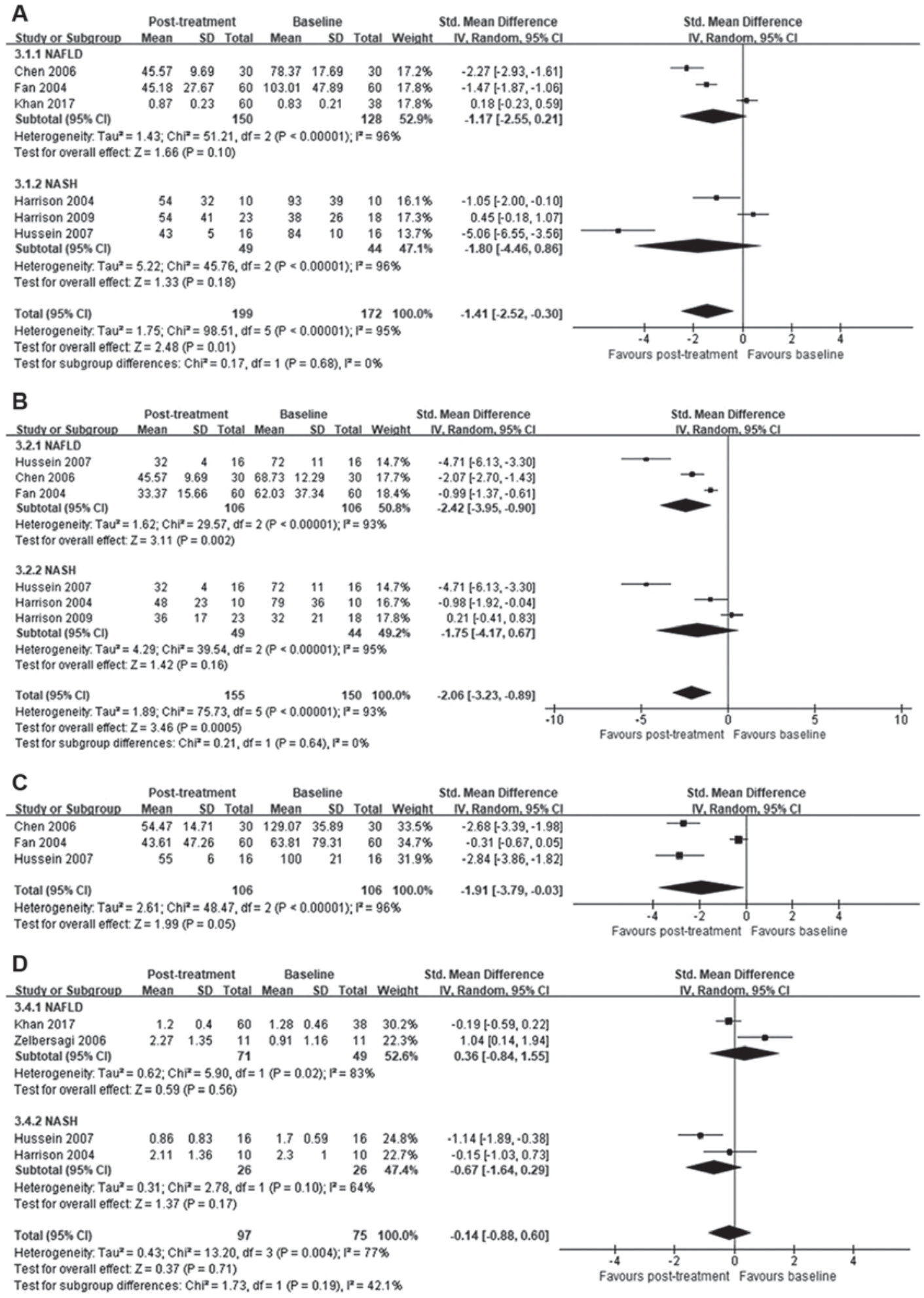

Figure 3. Forest plots illustrating improvement in biochemical and liver histological variables following treatment with orlistat. (A) Alanine aminotransferase; (B) aspartate aminotransferase; (C) $\gamma$-glutamyl transpeptidase; and (D) fibrosis score. NAFLD, non-alcoholic fatty liver; NASH, non-alcoholic steatohepatitis; SD, standard deviation; 95\% CI, 95\% confidence interval; IV, independent variable.

with NAFLD and NASH (SMD=-0.51; 95\% CI=-0.91 to -0.11 ; $\mathrm{P}=0.01)$. Between-study heterogeneity was moderate $\left(\mathrm{I}^{2}=31 \%\right.$; Fig. 4A).

Plasma triglycerides. The effect of orlistat on TG levels in five studies was analyzed. Overall, despite high heterogeneity $\left(\mathrm{I}^{2}=88 \%\right)$, TG levels were significantly reduced following orlistat treatment compared to baseline $(\mathrm{MD}=-0.93$;
95\% $\mathrm{CI}=-1.67$ to $-0.19 ; \mathrm{P}=0.01)$. Subgroup analysis determined a significant difference in patients with NAFLD (SMD $=-0.45$; 95\% CI $=-0.74$ to $-0.16 ; \mathrm{I}^{2}=28 \%$; $\mathrm{P}=0.002$ ), but not in patients with NASH (SMD $=-2.11 ; 95 \% \mathrm{CI}=-6.00$ to $1.79 ; \mathrm{I}^{2}=96 \%$; $\mathrm{P}=0.29$; Fig. 4B).

HOMA-IR. HOMA-IR levels were reported in four studies. Compared to baseline, orlistat treatment caused a modest, but 
A

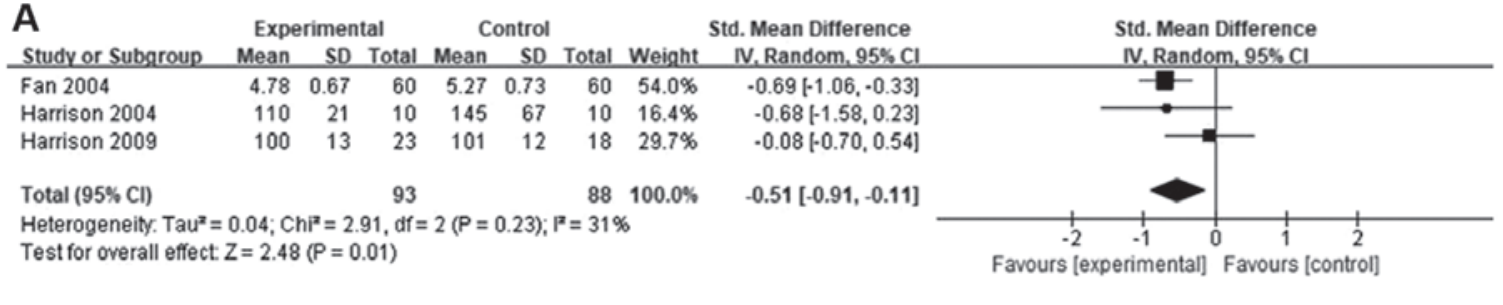

\section{B}

$\begin{array}{ccc}\text { Experimental Control } & \text { Std. Mean Difference } \\ \text { Mean SD Total Mean SD Total Weioht N. Random. } 95 \% \mathrm{CI}\end{array}$

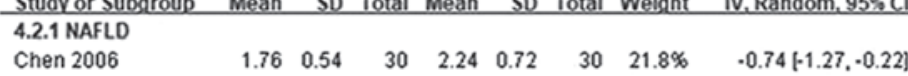

Fan 2004

Khan 2017

$\begin{array}{lllllll}1.63 & 0.79 & 60 & 2.02 & 0.77 & 60 & 23.1 \%\end{array}$

$\begin{array}{rrrrrrr}1.95 & 0.6 & 60 & 2.08 & 0.76 & 38 & 22.8 \% \\ & & 150\end{array}$

Subtotal $(95 \% \mathrm{Cl})$

Heterogeneity. $\operatorname{Tau}^{2}=0.02 ; \mathrm{Chi}^{2}=2.79, \mathrm{df}=2(P=0.25) ; \mathrm{P}^{2}=28 \%$

Test for overall effect: $Z=3.05(P=0.002)$

4.2.2 NASH

Harrison 2004
Hussein 2007

Subtotal $(95 \% \mathrm{Cl})$

$\begin{array}{lllll}147 & 53 & 10 & 158 & 8 \\ 163 & 10 & 16 & 238 & 23\end{array}$

Heterogeneity. $\mathrm{Tau}^{2}=7.59 ; \mathrm{Chi}^{2}=25.14$,

Test for overall effect: $Z=1.06(P=0.29)$

Total $(95 \% \mathrm{Cl})$

176

Test for overall effect $Z=2.46(P=0.01)$

Test for subgroup differences: $\mathrm{ChP}^{2}=0.69, \mathrm{df}=1(P=0.41), \mathrm{P}^{2}=0 \%$

$10 \quad 18.2 \%$

$16 \quad 14.1 \%$

$2632.3 \%$

$=1(P<0.00001) ; P=96 \%$

$54100.0 \%$
$-0.50[-0.86,-0.13]$

$-0.19[-0.60,0.21\}$

$-0.45[-0.74,-0.16]$

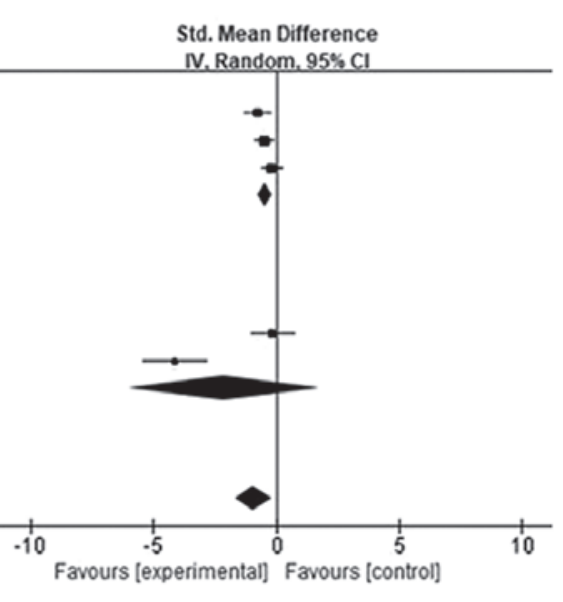

$-0.15[-1.03,0.73]$ $-4.12[-5.40,-2.84]$ $-2.11[-6.00,1.79]$

$-0.93[-1.67,-0.19]$

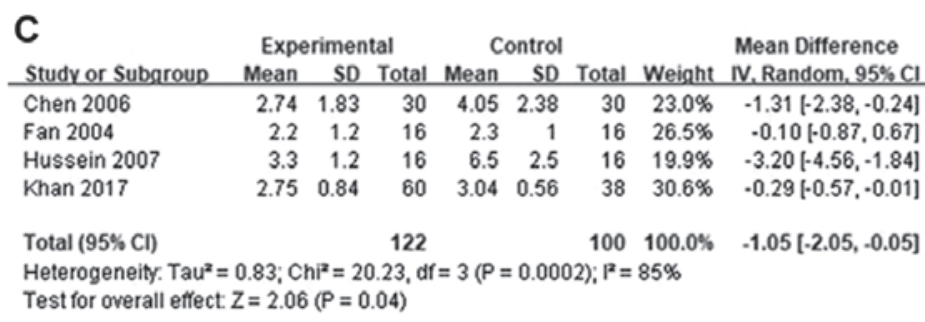

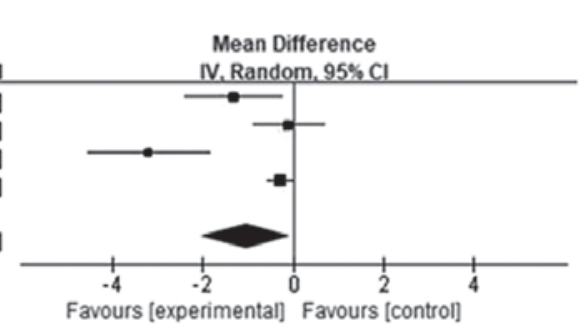

D Experimental Control Mean Difference

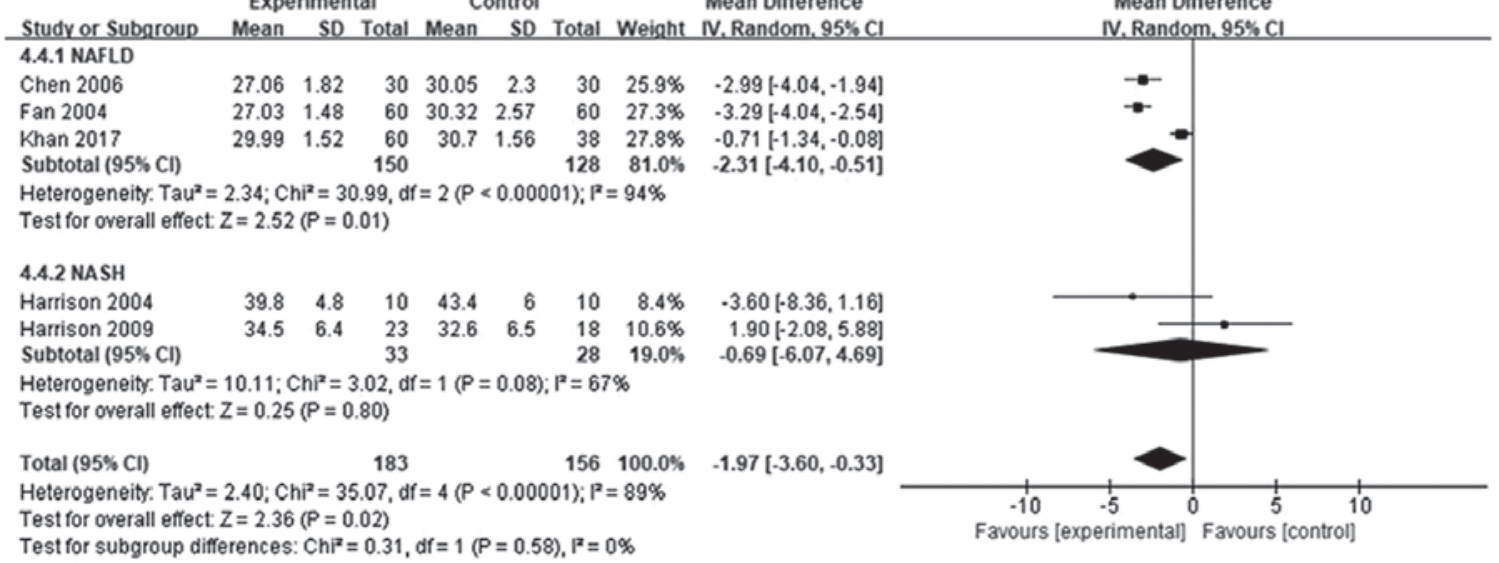

Figure 4. Forest plots illustrating improvement in biochemical and anthropometric variables. (A) Fasting glucose; (B) triglycerides; (C) homeostasis model assessment of insulin resistance; and (D) body mass index. NAFLD, non-alcoholic fatty liver; NASH, non-alcoholic steatohepatitis; SD, standard deviation; 95\% CI, 95\% confidence interval; IV, independent variable.

significant, reduction in insulin resistance $(\mathrm{MD}=-1.05 ; 95 \%$ $\mathrm{CI}=-2.05$ to $-0.05 ; \mathrm{I}^{2}=85 \%, \mathrm{P}=0.04$; Fig. 4C).

$B M I$. Data from five studies were used to evaluate BMI (Fig. 4D). Compared to baseline, orlistat treatment significantly reduced $\mathrm{BMI}(\mathrm{MD}=-1.97 ; 95 \% \mathrm{CI}=-3.60$ to -0.33 ; $\mathrm{P}=0.02)$, although between-study heterogeneity was high $\left(\mathrm{I}^{2}=89 \%\right)$. Subgroup analysis identified a significant difference in patients with NAFLD $(\mathrm{MD}=-2.31 ; 95 \% \mathrm{CI}=-4.10$ to -0.51 ;
$\left.\mathrm{I}^{2}=94 \% ; \mathrm{P}=0.01\right)$ but not in patients with NASH $(\mathrm{MD}=-0.69$; $95 \% \mathrm{CI}=-6.07$ to $\left.4.69 ; \mathrm{I}^{2}=67 \% ; \mathrm{P}=0.80\right)$.

Adverse events. Of the seven studies included in the meta-analysis, four reported adverse events $(11,17,22,24)$. Zelber-Sagi et al (17) reported that 2 patients withdrew from the study due to gastrointestinal side effects. Fan et al (22) reported that 2 patients withdrew from the study due to fecal urgency and fecal incontinence following 8 weeks of treatment. 
Mild gastrointestinal discomfort symptoms, including nausea, abdominal cramps, fecal urgency, fecal incontinence and increased defecation were also reported $(11,12,17,22,24)$. No serious treatment-related adverse effects on cardiovascular outcome or carcinogenesis were reported in any of the included studies.

\section{Discussion}

The present study to the best of our knowledge is the first systematic review and meta-analysis investigating the efficacy and safety of orlistat intervention in patients with NAFLD or NASH. Following a review of the literature, seven trials were identified that satisfied the inclusion criteria. Although the results of the current analysis are limited by the relatively small number of studies included, the data suggest improvements in biochemical, metabolic and anthropometric indicators of liver disease and in overall safety by orlistat treatment in patients with NAFLD or NASH.

In the studies analyzed, orlistat improved biochemical markers of liver disease. Orlistat treatment reduced the levels of ALT, AST and GGT compared to baseline in patients with NAFLD; however, the subgroup analysis did not reveal significant differences in patients with NASH. Aminotransferases are sensitive indicators of liver damage and cellular integrity and are mainly located in hepatocytes $(14,15,26)$. When hepatocytes are damaged, for example during liver disease, blood aminotransferase levels increase (15). It is considered that orlistat may reduce damage to hepatocytes by decreasing fat accumulation in the liver, and thereby decrease aminotransferase levels (23).

However, the effect of orlistat on liver histological variables remains unclear. Overall assessment of the four studies noting liver fibrosis score revealed no significant changes in the scores following treatment with orlistat compared to baseline. These results are consistent with those of Tilg and Moschen (27) who demonstrated that orlistat failed to improve the histopathology of NAFLD. Therefore, orlistat should not be considered as a medication for alleviating fibrosis in patients with NAFLD or NASH

As an anti-obesity drug, orlistat has been demonstrated to reduce postprandial lipid levels following high-fat meals. A study by Gabriel et al (28) identified that administration of orlistat suppressed the postprandial rise of TG levels in healthy adult volunteers following consumption of meals with $50 \%$ fat. Nakou et al (29) observed a greater decrease in TG levels with ezetimibe plus orlistat therapy compared with monotherapy with ezetimibe alone. In the current meta-analysis, TG levels were markedly improved following orlistat treatment compared to baseline, suggesting that orlistat may alleviate lipid accumulation, although statistical significance was not achieved in patients with NASH.

The present results also indicated that orlistat treatment reduced plasma glucose levels compared to baseline in patients with NAFLD and NASH. Kujawska-Łuczak et al (30) observed similar results while examining the effect of orlistat on glucose/ insulin homeostasis in obese premenopausal women. Orlistat has been further indicated to improve glycemic control and insulin resistance, as well as reduce body weight gain and BMI, in patients with obesity and type 2 diabetes $(31,32)$. Based on these data, the effect of orlistat on insulin resistance and BMI in patients with NAFLD was analyzed. HOMA-IR was used to evaluate insulin resistance and BMI substituted for incomplete data on body weight. As expected, improvement in BMI was associated with improved insulin resistance, and HOMA-IR scores and BMI were significantly lower following orlistat treatment compared to baseline.

Previous results have indicated that hepatic steatosis and inflammation may be improved by weight loss or reduction of BMI (33). However, Harrison et al (23) reported that no significant differences in hepatic steatosis and inflammation were found between orlistat/diet/vitamin $\mathrm{E}$ and diet/vitamin $\mathrm{E}$ groups. The limited information collected on this aspect in our included studies hampered analysis of hepatic steatosis, necroinflammation and ballooning. Of the included studies, only two reported on steatosis and inflammation $(23,24)$, and one on ballooning (23). Thus, further studies are required to investigate whether orlistat may improve liver histology.

Several limitations of the current analysis should be noted. First, a relatively small number of studies and sample sizes were included in the meta-analysis. Second, a high heterogeneity was observed, probably in part due to the small sample sizes, inconsistent duration of treatment, and lack of control groups, which prevented the evaluation of clinically significant conclusions. Third, only three studies investigated liver histological variables in patients with NAFLD and NASH.

In conclusion, the present systematic review and meta-analysis assessed the effect of the anti-obesity drug orlistat on various disease biomarkers in patients with NAFLD and NASH. The results suggest that, although orlistat does not consistently reverse liver fibrosis, it appears to improve other biochemical, metabolic and anthropometric indicators and to a certain extent, may contribute to prognosis. However, due to the limited sample size and high heterogeneity, these findings did not prove statistically significant in all subgroup analyses. Nonetheless, the data support the use of orlistat as a therapeutic strategy to improve biochemical indicators of liver damage, but not as a drug of choice for the management of NAFLD or NASH. Larger sample sizes and additional liver histological parameters (including hepatic steatosis, ballooning, necroinflammation and fibrosis) should be assessed over a longer follow-up period in future studies.

\section{Acknowledgements}

Not applicable.

\section{Funding}

The present study was supported by the National Natural Science Foundation of China (grant no. 81470303), the Top-notch Academic Programs Project of Jiangsu Higher Education Institutions (grant no. PPZY2015B161) and the Jiangsu Province Students Innovation and Entrepreneurship Training Program (grant no. 201710313022Z).

\section{Authors' contributions}

HW and YC conducted the meta-analysis including design and drafting of the manuscript. LW and ZX verified the data 
extracted following the literature search. HW, YL and JC were responsible for proofreading and revising the review critically for intellectual content. All authors read and approved the final manuscript.

\section{Availability of data and materials}

All data generated and/or analyzed during this study are included in this published article.

\section{Ethics approval and consent to participate}

Not applicable.

\section{Consent for publication}

Not applicable.

\section{Competing interests}

The authors declare that they have no competing interests.

\section{References}

1. Bellentani S, Scaglioni F, Marino M and Bedogni G: Epidemiology of non-alcoholic fatty liver disease. Dig Dis 28: 155-161, 2010.

2. Lazo M and Clark JM: The epidemiology of nonalcoholic fatty liver disease: A global perspective. Semin Liver Dis 28: 339-350, 2008.

3. Zhu JZ, Dai YN, Wang YM, Zhou QY, Yu CH and Li YM: Prevalence of nonalcoholic fatty liver disease and economy. Dig Dis Sci 60: 3194-3202, 2015.

4. Vanni E, Bugianesi E, Kotronen A, De Minicis S, Yki-Järvinen H and Svegliati-Baroni G: From the metabolic syndrome to NAFLD or vice versa? Dig Liver Dis 42: 320-330, 2010.

5. Li L, Liu DW, Yan HY, Wang ZY, Zhao SH and Wang B: Obesity is an independent risk factor for non-alcoholic fatty liver disease: Evidence from a meta-analysis of 21 cohort studies. Obes Rev 17: 510-519, 2016

6. Zhao ZH, Liu XL and Fan JG: Research on the natural history of non-alcoholic fatty liver disease should be taken seriously. Zhonghua Gan Zang Bing Za Zhi 25: 81-84, 2017 (In Chinese).

7. Bhala N, Angulo P, van der Poorten D, Lee E, Hui JM, Saracco G, Adams LA, Charatcharoenwitthaya P, Topping JH, Bugianesi E, et al: The natural history of nonalcoholic fatty liver disease with advanced fibrosis or cirrhosis: An international collaborative study. Hepatology 54: 1208-1216, 2011.

8. Duvnjak M, Tomasic V, Gomercic M, Smircic Duvnjak L, Barsic N and Lerotic I: Therapy of nonalcoholic fatty liver disease: Current status. J Physiol Pharmacol 60 (Suppl 7): 57-66, 2009.

9. Narayanaswami V and Dwoskin LP: Obesity: Current and potential pharmacotherapeutics and targets. Pharmacol Ther 170: 116-147, 2017.

10. Kushner RF: Medical management of obesity. Semin Gastrointest Dis 13: 123-132, 2002.

11. Ali Khan R, Kapur P, Jain A, Farah F and Bhandari U: Effect of orlistat on periostin, adiponectin, inflammatory markers and ultrasound grades of fatty liver in obese NAFLD patients. Ther Clin Risk Manag 13: 139-149, 2017.

12. Chen Z, Liang B, Wang Y and Fan X: The analysis of the efficacy and risk factors in obesity with non-alcoholic fatty liver disease subjects treated with orlistat. Chin J Clin Hepatol 22: 123-124, 2006 (In Chinese).

13. Harrison SA, Fincke C, Helinski D, Torgerson S and Hayashi P: A pilot study of orlistat treatment in obese, non-alcoholic steatohepatitis patients. Aliment Pharmacol Ther 20: 623-628, 2004

14. Fernández S and Córdoba M: Progesterone causes metabolic changes involving aminotransferases and creatine kinase in cryopreserved bovine spermatozoa. Anim Reprod Sci 164: 90-96, 2016.
15. Bruinsma BG, Wu W, Ozer S, Farmer A, Markmann JF, Yeh H and Uygun $\mathrm{K}$ : Warm ischemic injury is reflected in the release of injury markers during cold preservation of the human liver. PLoS One 10: e0123421, 2015

16. Filippatos TD, Derdemezis CS, Gazi IF, Nakou ES, Mikhailidis DP and Elisaf MS: Orlistat-associated adverse effects and drug interactions: A critical review. Drug Saf 31: 53-65, 2008.

17. Zelber-Sagi S, Kessler A, Brazowsky E, Webb M,Lurie Y, Santo M, Leshno M, Blendis L, Halpern Z and Oren R: A double-blind randomized placebo-controlled trial of orlistat for the treatment of nonalcoholic fatty liver disease. Clin Gastroenterol Hepatol 4: 639-644, 2006.

18. Moher D, Liberati A, Tetzlaff J and Altman DG; PRISMA Group: Preferred reporting items for systematic reviews and meta-analyses: The PRISMA statement. Int J Surg 8: 336-341, 2010.

19. Furlan AD, Pennick V, Bombardier C and van Tulder M; Editorial Board, Cochrane Back Review Group: 2009 updated method guidelines for systematic reviews in the Cochrane Back Review Group. Spine 34: 1929-1941, 2009.

20. Stang A: Critical evaluation of the Newcastle-Ottawa scale for the assessment of the quality of nonrandomized studies in meta-analyses. Eur J Epidemiol 25: 603-605, 2010.

21. Li Z, Xue J, Chen P, Chen L, Yan S and Liu L: Prevalence of nonalcoholic fatty liver disease in mainland of China: A meta-analysis of published studies. J Gastroenterol Hepatol 29: 42-51, 2014.

22. Fan Z, Qiu D, Xie W, Hu H, Chen Y, Sun Z, Liu S and Zeng M: Clinical study of obesity associated non-alcoholic fatty liver disease. Chin Hepatol 9: 155-158, 2004 (In Chinese).

23. Harrison SA, Fecht W, Brunt EM and Neuschwander-Tetri BA: Orlistat for overweight subjects with nonalcoholic steatohepatitis: A randomized, prospective trial. Hepatology 49: 80-86, 2009.

24. Hussein O, Grosovski M, Schlesinger S, Szvalb S and Assy N: Orlistat reverse fatty infiltration and improves hepatic fibrosis in obese patients with nonalcoholic steatohepatitis (NASH). Dig Dis Sci 52: 2512-2519, 2007.

25. Angulo P, Hui JM, Marchesini G, Bugianesi E, George J, Farrell GC, Enders F, Saksena S, Burt AD, Bida JP, et al: The NAFLD fibrosis score: A noninvasive system that identifies liver fibrosis in patients with NAFLD. Hepatology 45: 846-854, 2007.

26. Mahboub P, Ottens P, Seelen M, 't Hart N, Van Goor H, Ploeg R, Martins PN and Leuvenink H: Gradual rewarming with gradual increase in pressure during machine perfusion after cold static preservation reduces kidney ischemia reperfusion injury. PLoS One 11: e0152006, 2016

27. Tilg $\mathrm{H}$ and Moschen A: Weight loss: Cornerstone in the treatment of non-alcoholic fatty liver disease. Minerva Gastroenterol Dietol 56: 159-167, 2010.

28. Gabriel FS, Samson CE, Abejuela ZR, Sicat-Gabriel PR, Sumpio JP, Zacarias MB and Mercado-Asis LB: Postprandial effect of orlistat on the peaking of lipid level after sequential high fat meals. Int J Endocrinol Metab 10: 458-463, 2012.

29. Nakou ES, Filippatos TD, Agouridis AP, Kostara C, Bairaktari ET and Elisaf MS: The effects of ezetimibe and/or orlistat on triglyceride-rich lipoprotein metabolism in obese hypercholesterolemic patients. Lipids 45: 445-450, 2010.

30. Kujawska-Łuczak M, Musialik K, Szulińska M, Swora-CwynarE, Kargulewicz A, Grzymisławska M, Pupek-Musialik D and Bogdański P: The effect of orlistat versus metformin on body composition and insulin resistance in obese premenopausal women: 3-month randomized prospective open-label study. Arch Med Sci 13: 725-731, 2017.

31. Scheen AJ and Van Gaal LF: Combating the dual burden: Therapeutic targeting of common pathways in obesity and type 2 diabetes. Lancet Diabetes Endocrinol 2: 911-922, 2014.

32. Smith-Marsh D: Pharmacological strategies for preventing type 2 diabetes in patients with impaired glucose tolerance. Drugs Today (Barc) 49: 499-507, 2013.

33. Chalasani N, Younossi Z, Lavine JE, Diehl AM, Brunt EM, Cusi K, Charlton M and Sanyal AJ; American Gastroenterological Association; American Association for the Study of Liver Diseases; American College of Gastroenterologyh: The diagnosis and management of non-alcoholic fatty liver disease: Practice guideline by the American Gastroenterological Association, American Association for the Study of Liver Diseases, and American College of Gastroenterology. Gastroenterology 142: 1592-1609, 2012 